\title{
Capacidade de cultivares de arroz de modificar o pH de soluções nutritivas na presença de alumínio
}

\author{
Renilton Joaquim de Mendonça ${ }^{(1)}$, José Cambraia ${ }^{(2)}$, Marco Antonio Oliva ${ }^{(2)}$ e Juraci Alves de Oliveira(2)
}

(1)Rua 05, Quadra 06, Lote 15, Setor do Lago, CEP 76550-000 Porangatu, GO. E-mail: rjmendonca@yahoo.com.br(2)Universidade Federal de Viçosa, Dep. de Biologia Geral, Avenida P.H. Rolfs, s/no, CEP 36571-000 Viçosa, MG. E-mail: cambraia@ufv.br, moliva@ufv.br, jalves@ufv.br

\begin{abstract}
Resumo - O objetivo deste trabalho foi avaliar o efeito do alumínio sobre a capacidade das cultivares de arroz Fernandes (tolerante) e Maravilha (sensível) de modificar o pH de soluções nutritivas com diferentes proporções de $\mathrm{NO}_{3}{ }^{-} / \mathrm{NH}_{4}{ }^{+}$. Após exposição das plantas ao $\mathrm{Al}(0 \mathrm{mM}$ e 1,5 mM), durante 15 dias, foram determinados: quantidade de prótons exsudados, alongamento, produção de matéria seca e teor de Al nas raízes e folhas. $\mathrm{O}$ alongamento e produção de matéria seca, de raízes e folhas, se reduziram em presença de Al, independentemente da proporção $\mathrm{NO}_{3}{ }^{-} / \mathrm{NH}_{4}{ }^{+}$, e essas reduções se intensificaram com o aumento na concentração de $\mathrm{NH}_{4}^{+}$. Os teores de $\mathrm{Al}$ aumentaram nas raízes e folhas, com aumento na concentração de $\mathrm{NH}_{4}^{+}$na solução nutritiva. Houve forte alcalinização da solução nutritiva com nitrogênio exclusivamente na forma de $\mathrm{NO}_{3}^{-}$, que se reduziu em presença de $\mathrm{Al}$. Em soluções nutritivas com $\mathrm{NH}_{4}{ }^{+}$, ao contrário, observou-se acidificação, que se intensificou com aumento na concentração de $\mathrm{NH}_{4}{ }^{+}$. A cultivar tolerante sempre consumiu mais prótons da solução nutritiva com $\mathrm{N}$ exclusivamente na forma de $\mathrm{NO}_{3}{ }^{-}$, e menos prótons daquela que continha $\mathrm{NH}_{4}{ }^{+}$, independentemente da proporção $\mathrm{NO}_{3}{ }^{-} / \mathrm{NH}_{4}{ }^{+}$. Essa cultivar foi, portanto, capaz de ajustar seu balanço de prótons e modificou o pH para valores que favoreciam menor absorção e maior tolerância ao alumínio.
\end{abstract}

Termos para indexação: Oryza sativa, íon amônio, nitrato, tolerância ao Al, toxidez de Al.

\section{Rice cultivars ability to change nutrient solution $\mathrm{pH}$ in the presence of aluminum}

\begin{abstract}
The objective of this work was to evaluate aluminum effect on the ability of two rice cultivars, Fernandes (tolerant) and Maravilha (sensitive), to change the $\mathrm{pH}$ of nutrient solutions with different $\mathrm{NO}_{3}{ }^{-} / \mathrm{NH}_{4}{ }^{+}$ ratios. After plant exposure to $0 \mathrm{mM}$ and $1.5 \mathrm{mM} \mathrm{Al}$ for 15 days, the amount of exudated protons, elongation, dry matter yield and Al content of roots and leaves were determined. Elongation and dry matter yield of roots and leaves reduced in the presence of $\mathrm{Al}$ independently of $\mathrm{NO}_{3}{ }^{-} / \mathrm{NH}_{4}{ }^{+}$ratio, and this reduction was intensified with increasing of $\mathrm{NH}_{4}^{+}$concentration. Aluminum contents increased in roots and leaves with increasing of $\mathrm{NH}_{4}^{+}$ concentration in nutrient solution. An alkalization of the nutrient solution containing $\mathrm{N}$ exclusively in $\mathrm{NO}_{3}{ }^{-}$form was observed, but this alkalization decreased with $\mathrm{Al}$ treatment. In nutrient solutions containing $\mathrm{NH}_{4}{ }^{+}$, on the contrary, an acidification was observed, which intensified with the increase of $\mathrm{NH}_{4}{ }^{+}$concentration. The tolerant cultivar always consumed more protons of the nutrient solution containing $\mathrm{N}$ exclusively in the $\mathrm{NO}_{3}{ }^{-}$form and less protons of that containing $\mathrm{NH}_{4}{ }^{+}$, independently of the $\mathrm{NO}_{3}{ }^{-} / \mathrm{NH}_{4}{ }^{+}$ratio. This cultivar, therefore, showed higher ability to adjust its protons balance, changing the $\mathrm{pH}$ to values that favored lower $\mathrm{Al}$ uptake and higher tolerance to aluminum.
\end{abstract}

Index terms: Oryza sativa, nitrate, ammonium, Al tolerance, Al toxicity.

\section{Introdução}

A tolerância das plantas ao alumínio tem sido, muitas vezes, associada com a capacidade que elas têm de alterar o pH na rizosfera (Antunes \& Nunes, 1997; Degenhardt et al., 1998). De acordo com essa hipótese, as plantas tolerantes seriam capazes de elevar o $\mathrm{pH}$ da rizosfera e, assim, reduzir a solubilidade e a absorção do Al, aliviando, conseqüentemente, os efeitos fitotóxicos desse íon (Silva et al., 2002). Essa capacidade tem sido encontrada em várias espécies de importância econô- mica, inclusive em algumas cultivares de arroz de origem asiática (Ganesan et al, 1993; Sivaguru \& Paliwal, 1993).

A capacidade das plantas de modificar o $\mathrm{pH}$ da rizosfera, além da natureza específica de cada espécie ou cultivar, depende de vários fatores do ambiente, entre os quais a nutrição nitrogenada (Bernardo et al., 1984). Quando o nitrogênio é suprido exclusivamente na forma nítrica, as plantas tendem a elevar o $\mathrm{pH}$ do meio utilizado, em razão de esse ânion ser absorvido predominantemente por um simporte tipo $\mathrm{H}^{+} / \mathrm{NO}_{3}{ }^{-}$, que 
retira prótons do meio externo (Antunes \& Nunes, 1997; Tischner, 2000). Quando o N é suprido exclusivamente na forma amoniacal, o íon $\mathrm{NH}_{4}{ }^{+}$é absorvido por um canal de cátions, sem a absorção concomitante de prótons e, portanto, resulta no abaixamento do $\mathrm{pH}$ no meio externo (Marschner, 1995; Antunes \& Nunes, 1997).

Essa interrelação do $\mathrm{Al}$ com o metabolismo do $\mathrm{N}$ e as mudanças do $\mathrm{pH}$ na rizosfera, entretanto, parece ser bem mais complexa. No trigo, a tolerância das plantas ao $\mathrm{Al}$ parece estar ligada à capacidade de elas utilizarem nitrato na presença de amônia, e de aumentarem o pH do meio de crescimento (Taylor \& Foy, 1985). No triticale, entretanto, as mudanças do $\mathrm{pH}$ não foram relevantes ao mecanismo de tolerância ao $\mathrm{Al}$, e o íon $\mathrm{NH}_{4}{ }^{+}$teve efeito protetor contra a toxidez do $\mathrm{Al}$ (Antunes \& Nunes, 1997). No sorgo, em presença de Al, as plantas diminuíram o $\mathrm{pH}$ do meio, e o efeito foi mais forte nas soluções nutritivas em que a proporção $\mathrm{NO}_{3}{ }^{-} / \mathrm{NH}_{4}{ }^{+}$ decresceu (Cambraia et al., 1987). No arroz, foi verificado que a cultivar tolerante além de elevar o $\mathrm{pH}$ da solução nutritiva, mais do que a sensível, foi capaz de metabolizar o nitrato com maior eficiência em presença de Al (Ganesan et al., 1993; Sivaguru \& Paliwal, 1993).

Como o mecanismo de tolerância ao $\mathrm{Al}$ em arroz é ainda pouco conhecido, especialmente em cultivares nacionais, e há grande possibilidade de a nutrição nitrogenada estar intimamente relacionada com essa tolerância ao Al, resolveu-se realizar a presente pesquisa.

O objetivo deste trabalho foi avaliar o efeito do $\mathrm{Al}$ sobre a capacidade de duas cultivares de arroz, Fernandes (tolerante) e Maravilha (sensível), de modificar o $\mathrm{pH}$ de soluções nutritivas com diferentes proporções de $\mathrm{NO}_{3}{ }^{-} / \mathrm{NH}_{4}{ }^{+}$.

\section{Material e Métodos}

Foram utilizadas duas cultivares de arroz (Oryza sativa L.), cedidas pela Embrapa Arroz e Feijão: uma tolerante (Fernandes: CNA-1158) e outra sensível (Maravilha: CNA-6843-1) ao Al (Fageria \& Carvalho, 1982; Fageria et al., 1988).

As sementes, selecionadas quanto ao tamanho e forma, foram tratadas com $\mathrm{H}_{2} \mathrm{SO}_{4}$ a $50 \%$, por 15 min, lavadas, em água corrente, esterilizadas superficialmente com hipoclorito de sódio a 2\% por 15 min e lavadas, novamente, em água corrente e em água desmineralizada. A seguir, foram colocadas para germinar em cartuchos de papel "germitest" (pH neutro), mergulhados em vasos de plástico com 1,6 L de solução nutritiva de Clark (Clark, 1975), pH 4, com um quinto de força iônica, sob aeração contínua.

As plântulas, após seleção quanto à uniformidade de tamanho e forma, foram transplantadas para vasos de polietileno, pintados externamente de preto, com 1,6 L de solução nutritiva de Clark, pH 4 (Clark, 1975), modificada para fornecer o $\mathrm{N}$ recomendado $\left(8 \mathrm{mM} \mathrm{L}^{-1}\right)$ : $100 \%$ na forma de $\mathrm{NO}_{3}{ }^{-}(100 / 0) ; 87,5 \%$ na forma de $\mathrm{NO}_{3}{ }^{-}$e $12,5 \%$ na forma de $\mathrm{NH}_{4}{ }^{+}(87,5 / 12,5) ; 50 \%$ na forma de $\mathrm{NO}_{3}{ }^{-}$e $50 \%$ na forma de $\mathrm{NH}_{4}{ }^{+}(50 / 50)$ e $100 \%$ na forma de $\mathrm{NH}_{4}{ }^{+}(0 / 100)$. $\mathrm{O} \mathrm{Al}$ foi adicionado a essas soluções nas concentrações de 0 e $1,50 \mathrm{mM}$, na forma de $\mathrm{Al}_{2}\left(\mathrm{SO}_{4}\right)_{3} \cdot 18 \mathrm{H}_{2} \mathrm{O}$.

A quantidade de prótons $\left(\mathrm{H}^{+}\right)$liberados ou removidos da solução nutritiva pelas plantas foi determinada por titulação, com $\mathrm{HCl}$ 0,1 N ou NaOH 0,1 N padronizados, ajustando-se diariamente o $\mathrm{pH}$ da solução nutritiva para 4 , a $25^{\circ} \mathrm{C}$. As soluções nutritivas foram renovadas a cada sete dias, e após 15 dias de cultivo o experimento foi encerrado. As plantas foram, então, colhidas, lavadas em água corrente e enxaguadas em água desmineralizada. As raízes foram lavadas em água corrente, depois lavadas com $\mathrm{HCl}$ 0,1 N por 15 min, lavadas novamente em água corrente e, finalmente, enxaguadas em água desmineralizada. Determinou-se o comprimento da raiz seminal, ou seja, da maior raiz, e da parte aérea, e a massa da matéria seca das duas partes das plantas, após secadas em estufa a $70^{\circ} \mathrm{C}$, até peso constante.

Após mineralização das amostras com uma mistura nítrico-perclórica, os teores de $\mathrm{Al}$ foram determinados pelo método da aluminona (Wang \& Wood, 1973)

A obtenção das plântulas e o experimento foram conduzidos em sala de crescimento, com temperatura controlada $\left(25 \pm 1^{\circ} \mathrm{C}\right)$, fluxo de fótons fotossintéticos de $215 \mu \mathrm{mol} \mathrm{m} \mathrm{m}^{-2} \mathrm{~s}^{-1} \mathrm{e}$ fotoperíodo de 16 horas.

O experimento foi disposto segundo esquema fatorial 2x2x4x3 (duas cultivares, dois níveis de Al, quatro proporções $\mathrm{NO}_{3}{ }^{-} / \mathrm{NH}_{4}{ }^{+}$e três repetições), em delineamento inteiramente casualizado, com três repetições. Os resultados foram submetidos à análise de variância e as médias comparadas pelo teste de Tukey a 5\% de probabilidade. 


\section{Resultados e Discussão}

Os comprimentos da raiz seminal, ou da maior raiz, e o comprimento da parte aérea, nas duas cultivares de arroz, independentemente da proporção $\mathrm{NO}_{3}{ }^{-} / \mathrm{NH}_{4}{ }^{+}$, se reduziram na presença do $\mathrm{Al}$ (Tabela 1). Por outro lado, independentemente da presença de $\mathrm{Al}$, à medida em que se reduziu a proporção $\mathrm{NO}_{3}{ }^{-} / \mathrm{NH}_{4}{ }^{+}$na solução nutritiva, também se observou redução nos comprimentos das raízes e da parte aérea, nas duas cultivares. Santamaria et al. (1999) também encontraram correlação linear e positiva, entre o comprimento radicular de algumas culturas e a concentração de $\mathrm{NO}_{3}{ }^{-}$na solução nutritiva. Grauer \& Horst (1990) obtiveram resultados semelhantes com plantas de centeio, sob condições similares, e atribuíram a severa inibição do alongamento radicular à forte toxicidade dos íons $\mathrm{H}^{+}$acumulados no meio de cultivo, na medida em que se aumentou a concentração de $\mathrm{NH}_{4}{ }^{+}$. Aparentemente, isto acontece porque na presença dessa fonte de $\mathrm{N}$ há uma diminuição na atividade do simporte $\mathrm{H}^{+} / \mathrm{NO}_{3}{ }^{-}$, o que resulta em acúmulo de $\mathrm{H}^{+}$no meio de cultivo (Marschner, 1995). Paralelamente a essa redução no comprimento da maior raiz, com a diminuição na proporção $\mathrm{NO}_{3}{ }^{-} / \mathrm{NH}_{4}{ }^{+}$da solução nutritiva, observou-se maior acúmulo de $\mathrm{Al}$ nas raízes das duas cultivares, principalmente no tratamento em que o $\mathrm{NH}_{4}{ }^{+}$foi a única fonte de nitrogênio (Tabela 1). Correlações negativas, entre taxa de alongamento radicular e a concentração de $\mathrm{Al}$ nas raízes, têm sido observadas em outras culturas como o sorgo (Cambraia et al., 1991) e o milho (Cambraia \& Cambraia, 1995), o que indica que não apenas o acúmulo de $\mathrm{H}^{+}$(Grauer \& Horst, 1990), mas também a maior absorção de Al compromete o crescimento radicular.

O comprimento da maior raiz e da parte aérea da cultivar tolerante foi quase sempre maior do que da cultivar sensível - independentemente da presença de $\mathrm{Al}$ ou da proporção $\mathrm{NO}_{3}{ }^{-} / \mathrm{NH}_{4}{ }^{+}$-, exceto na ausência de $\mathrm{Al}$ e com o $\mathrm{N}$ fornecido exclusivamente na forma amoniacal, na qual não se observou diferença significativa entre as cultivares. As reduções no comprimento da maior raiz e da parte aérea, causadas pela presença do $\mathrm{Al}$ na solução nutritiva, foram, de modo geral, mais intensas na cultivar sensível, para todas as proporções $\mathrm{NO}_{3}{ }^{-} / \mathrm{NH}_{4}{ }^{+}$da solução nutritiva.

As reduções de matéria seca, tanto das raízes quanto da parte aérea, causadas pelo $\mathrm{Al}$, à semelhança do que aconteceu quanto ao comprimento das raízes e da parte aérea, tenderam a se intensificar com o aumento da concentração de $\mathrm{NH}_{4}^{+}$na solução nutritiva, principalmente nas raízes da cultivar sensível (Tabela 1).

No presente trabalho, foram observados resultados semelhantes aos de Smith et al. (1990), Santamaria et al. (1999) e Traore \& Maranville (1999), que constataram redução na produção de matéria seca de milheto, sorgo e beterraba, respectivamente, na medida em que se reduziu a proporção $\mathrm{NO}_{3}{ }^{-} / \mathrm{NH}_{4}{ }^{+}$na solução nutritiva.

Tabela 1. Efeito do Al sobre o comprimento, a produção de matéria seca e os teores de Al em duas cultivares de arroz, cultivadas em soluções nutritivas, com diferentes proporções de $\mathrm{NO}_{3}{ }^{-} / \mathrm{NH}_{4}{ }^{+(1)}$.

\begin{tabular}{|c|c|c|c|c|c|c|c|}
\hline \multirow[t]{2}{*}{$\mathrm{Al}(\mathrm{mM})$} & \multirow{2}{*}{$\begin{array}{c}\text { Proporção } \\
\mathrm{NO}_{3}^{-} / \mathrm{NH}_{4}{ }^{+} \\
\end{array}$} & \multicolumn{2}{|c|}{ Comprimento (cm) } & \multicolumn{2}{|c|}{ Matéria seca $(\mathrm{g})$} & \multicolumn{2}{|c|}{ Teor de alumínio $\left(\mathrm{mg} \mathrm{g}^{-1}\right)$} \\
\hline & & Raízes & Parte aérea & Raízes & Parte aérea & Raízes & Parte aérea \\
\hline & & \multicolumn{6}{|c|}{ Fernandes (tolerante) } \\
\hline \multirow{3}{*}{0,0} & $100 / 0$ & $30,7 \mathrm{Aa}$ & $36,9 \mathrm{Aa}$ & $0,21 \mathrm{Aa}$ & $0,47 \mathrm{Aa}$ & $0,34 \mathrm{Bb}$ & $0,06 \mathrm{Bb}$ \\
\hline & $50 / 50$ & $28,1 \mathrm{Ba}$ & $33,1 \mathrm{Aa}$ & $0,11 \mathrm{Ca}$ & $0,42 \mathrm{ABa}$ & $0,51 \mathrm{ABb}$ & $0,11 \mathrm{Bb}$ \\
\hline & $0 / 100$ & $24,3 \mathrm{Ca}$ & $29,1 \mathrm{Ba}$ & $0,07 \mathrm{Da}$ & $0,31 \mathrm{Ba}$ & $0,64 \mathrm{Ab}$ & $0,16 \mathrm{Ab}$ \\
\hline \multirow[t]{4}{*}{1,5} & $100 / 0$ & $28,5 \mathrm{Ab}$ & $31,0 \mathrm{Ab}$ & $0,19 \mathrm{Ab}$ & $0,37 \mathrm{Ab}$ & $2,09 \mathrm{Da}$ & $0,18 \mathrm{Ba}$ \\
\hline & $87,5 / 12,5$ & $25,2 \mathrm{Bb}$ & $30,5 \mathrm{Ab}$ & $0,16 \mathrm{Ba}$ & $0,34 \mathrm{ABb}$ & $2,59 \mathrm{Ca}$ & $0,22 \mathrm{Ba}$ \\
\hline & $50 / 50$ & $21,0 \mathrm{Cb}$ & $25,6 \mathrm{Bb}$ & $0,09 \mathrm{Cb}$ & $0,24 \mathrm{Bb}$ & $3,13 \mathrm{Ba}$ & $0,32 \mathrm{Aa}$ \\
\hline & $0 / 100$ & $19,0 \mathrm{Cb}$ & $24,3 \mathrm{Bb}$ & $0,06 \mathrm{Da}$ & $0,23 \mathrm{Ba}$ & $3,78 \mathrm{Aa}$ & $0,33 \mathrm{Aa}$ \\
\hline \multicolumn{8}{|c|}{ Maravilha (sensível) } \\
\hline \multirow[t]{4}{*}{0,0} & $100 / 0$ & $27,4 \mathrm{Aa}$ & $32,2 \mathrm{Aa}$ & $0,15 \mathrm{Aa}$ & $0,30 \mathrm{Aa}$ & $0,36 \mathrm{Bb}$ & $0,08 \mathrm{Cb}$ \\
\hline & $87,5 / 12,5$ & $24,7 \mathrm{Ba}$ & $29,5 \mathrm{ABa}$ & $0,10 \mathrm{Ba}$ & $0,23 \mathrm{Aa}$ & $0,40 \mathrm{Bb}$ & $0,12 \mathrm{Cb}$ \\
\hline & $50 / 50$ & $22,7 \mathrm{Ba}$ & $28,7 \mathrm{ABa}$ & $0,08 \mathrm{Ba}$ & $0,20 \mathrm{Aa}$ & $0,88 \mathrm{Ab}$ & $0,40 \mathrm{Bb}$ \\
\hline & $0 / 100$ & $23,0 \mathrm{Ba}$ & $27,4 \mathrm{Ba}$ & $0,07 \mathrm{Ba}$ & $0,21 \mathrm{Aa}$ & $0,90 \mathrm{Ab}$ & $0,60 \mathrm{Ab}$ \\
\hline \multirow[t]{3}{*}{1,5} & $100 / 0$ & $19,5 \mathrm{Ab}$ & $21,0 \mathrm{Ab}$ & $0,08 \mathrm{Ab}$ & $0,13 \mathrm{Ab}$ & $2,98 \mathrm{Ca}$ & $0,70 \mathrm{Da}$ \\
\hline & $87,5 / 12,5$ & $19,5 \mathrm{Ab}$ & $18,5 \mathrm{ABb}$ & $0,05 \mathrm{Bb}$ & $0,12 \mathrm{Ab}$ & $3,20 \mathrm{Ba}$ & $0,80 \mathrm{Ca}$ \\
\hline & $0 / 100$ & $15,7 \mathrm{Bb}$ & $14,5 \mathrm{Cb}$ & $0,03 \mathrm{Bb}$ & $0,10 \mathrm{Ab}$ & $4,00 \mathrm{Aa}$ & $1,12 \mathrm{Aa}$ \\
\hline
\end{tabular}

(1)Médias, nas colunas, seguidas pela mesma letra maiúscula entre as proporções de $\mathrm{NO}_{3}{ }^{-} / \mathrm{NH}_{4}{ }^{+}$, para cada nível de $\mathrm{Al}$, e minúscula entre níveis de $\mathrm{Al}$, para cada proporção de $\mathrm{NO}_{3}{ }^{-} / \mathrm{NH}_{4}{ }^{+}$, na mesma cultivar, não diferem entre si a $5 \%$ de probabilidade, pelo teste de Tukey. 
As cultivares de arroz sofreram reduções que variaram de $10 \%$ a $57 \%$, na produção de matéria seca das raízes, e de $21 \%$ a $57 \%$ na produção de matéria seca da parte aérea, causadas pelo alumínio.

A cultivar tolerante apresentou, geralmente, maior produção de matéria seca nas raízes e na parte aérea, em relação à cultivar sensível, independentemente da proporção $\mathrm{NO}_{3}{ }^{-} / \mathrm{NH}_{4}{ }^{+}$e da presença de $\mathrm{Al}$, exceto quanto à produção de matéria seca das raízes, na ausência de $\mathrm{Al}$, e com o $\mathrm{N}$ fornecido exclusivamente na forma amoniacal. As reduções na produção de matéria seca das raízes e da parte aérea, causadas pelo $\mathrm{Al}$, foram sempre mais elevadas na cultivar sensível do que na tolerante, independentemente da proporção $\mathrm{NO}_{3}{ }^{-} / \mathrm{NH}_{4}{ }^{+}$.

Os teores de $\mathrm{Al}$, de modo geral, foram maiores nas plantas cultivadas em soluções nutritivas que possuíam $\mathrm{NH}_{4}{ }^{+}$em sua constituição e aumentaram com a redução da proporção $\mathrm{NO}_{3}{ }^{-} / \mathrm{NH}_{4}{ }^{+}$, nas duas partes das plantas das duas cultivares, independentemente da presença desse elemento nos tratamentos (Tabela 1). $\mathrm{Na}$ presença de $\mathrm{Al}$, como acontece com outras gramíneas como sorgo (Cambraia et al., 1987, 1991), milho (Cambraia \& Cambraia, 1995) e outras cultivares de arroz (Fageria \& Carvalho, 1982), os teores nas raízes foram sempre muito mais elevados do que na parte aérea, independentemente da nutrição nitrogenada. A cultivar sensível, em solução nutritiva contendo o $\mathrm{N}$ exclusivamente na forma amoniacal, nas plantas tratadas com $\mathrm{Al}$, apresentou teor desse elemento 3,4 vezes mais elevado do que a cultivar tolerante. Esse resultado indica que a adubação com sais de $\mathrm{NH}_{4}{ }^{+}$, ao invés de aliviar a toxicidade do $\mathrm{Al}$, como sugerem Antunes \& Nunes (1997) para triticale, pode aumentar a intensidade dos efeitos tóxicos do $\mathrm{Al}$ no arroz.

Em solução nutritiva contendo o $\mathrm{N}$ exclusivamente na forma nítrica, observou-se, na ausência de Al, forte alcalinização do meio de cultivo, pelas duas cultivares. A elevação mais intensa ocorreu na cultivar tolerante (Figura 1). Em presença de Al, a alcalinização do meio reduziu-se acentuadamente, passando para um valor médio aproximado de $13 \%$ daquele observado nos controles (ausência de $\mathrm{Al}$ ), nas duas cultivares. À medida que se adicionou e aumentou a concentração de $\mathrm{NH}_{4}{ }^{+}$ no meio de cultivo, observou-se tendência das plantas das duas cultivares de liberarem $\mathrm{H}^{+}$para a solução nutritiva. Isso se deve ao fato de que, quando expostas às soluções nutritivas que contêm $\mathrm{NH}_{4}{ }^{+}$, qualquer que seja sua proporção em relação ao $\mathrm{NO}_{3}{ }^{-}$, as plantas passam a absorver preferencialmente aquele cátion, até seu es- gotamento completo, ou quase completo, da solução nutritiva (Fleming, 1983; Bernardo et al., 1984). Segundo esses mesmos autores, em soluções nutritivas com as duas formas de $\mathrm{N}$, no início observa-se uma queda no $\mathrm{pH}$ da solução nutritiva até que todo o $\mathrm{NH}_{4}{ }^{+}$seja consumido e, a partir daí, o pH da solução nutritiva começa a se elevar. No presente trabalho, a fase de elevação no $\mathrm{pH}$ não foi observada, talvez porque o tempo de tratamento das plantas não tenha sido suficiente para chegar ao esgotamento do $\mathrm{NH}_{4}{ }^{+}$presente no meio de cultivo. Essa preferência pela absorção de $\mathrm{NH}_{4}{ }^{+}$, comparativamente à de $\mathrm{NO}_{3}{ }^{-}$, deve-se, provavelmente, ao fato de esse cátion ser absorvido por meio de canal iônico, sem gasto energético direto, enquanto a absor-

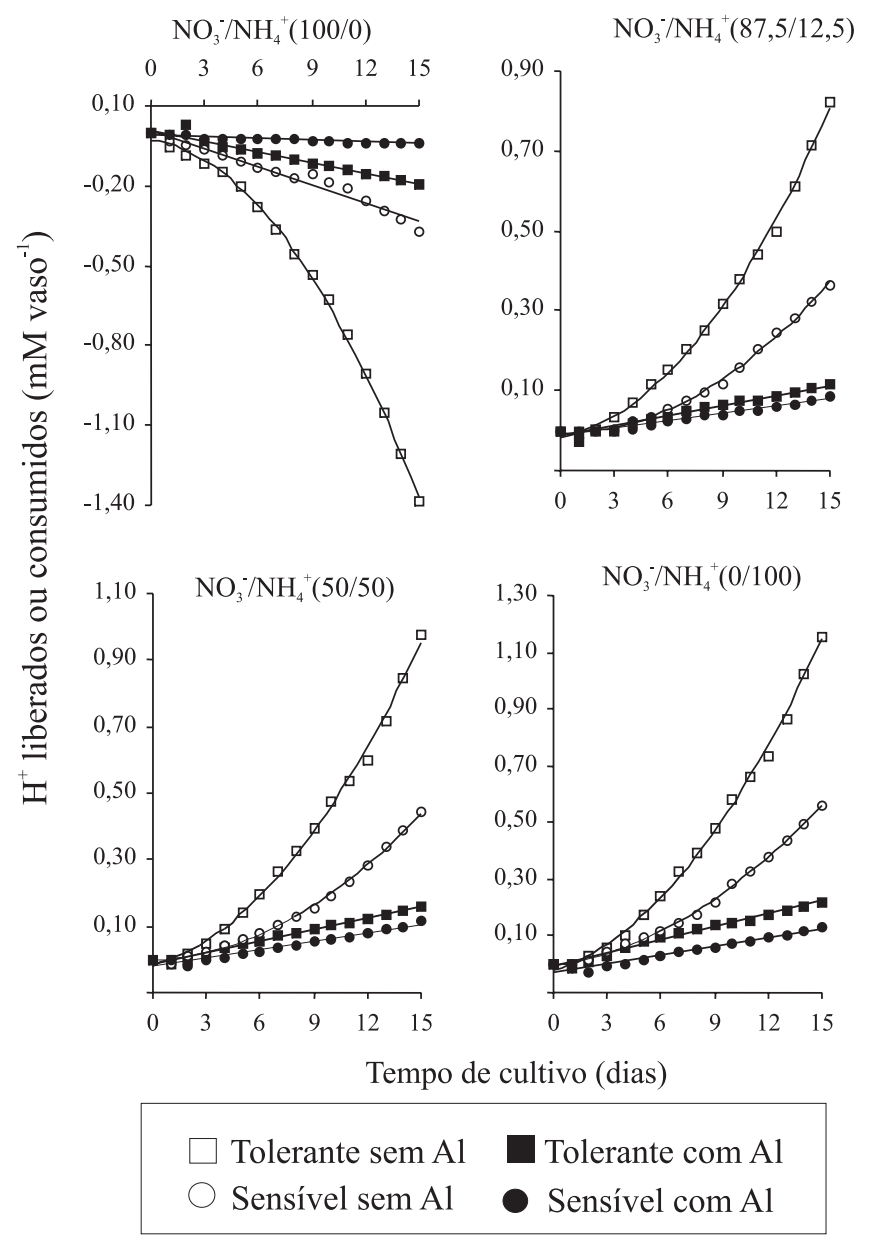

Figura 1. Liberação ou consumo de prótons $\left(\mathrm{H}^{+}\right)$de soluções nutritivas com diferentes proporções de $\mathrm{NO}_{3}{ }^{-} / \mathrm{NH}_{4}{ }^{+}$, por duas cultivares de arroz, na ausência ou presença de alumínio. 
ção de $\mathrm{NO}_{3}{ }^{-}$, usualmente, requer quantidade apreciável de energia. Como nesse caso não há retorno de quantidade proporcional de $\mathrm{H}^{+}$ao simplasma, caso do simporte $\mathrm{H}^{+} / \mathrm{NO}_{3}{ }^{-}$, ocorrerá acúmulo de $\mathrm{H}^{+}$no meio de cultivo, o que causará a queda do $\mathrm{pH}$ da solução nutritiva (Marschner, 1995; Santamaria et al., 1999).

A cultivar tolerante, quando exposta a $100 \%$ de $\mathrm{NO}_{3}{ }^{-}$, elevou mais o $\mathrm{pH}$, isto é, retirou mais prótons da solução nutritiva do que a cultivar sensível. A maior elevação do $\mathrm{pH}$, causada por essa cultivar, parece ter ocorrido pela sua maior capacidade de absorver $\mathrm{NO}_{3}{ }^{-}$, mediada pelo simporte $\mathrm{H}^{+} / \mathrm{NO}_{3}{ }^{-}$(Tischner, 2000), conforme sugere Ganesan et al. (1993). Essa mesma cultivar, quando cultivada em solução nutritiva contendo $\mathrm{NH}_{4}{ }^{+}$, em qualquer proporção ou, até mesmo, sendo a única fonte de N, foi, também, a que liberou mais prótons para a solução nutritiva. Como as plantas dessa cultivar produziram mais matéria seca, tanto nas raízes quanto na parte aérea, e desenvolveram um sistema radicular maior e com raízes mais compridas (Tabela 1 ), seria possível que essa maior capacidade de consumir ou liberar prótons fosse resultante de seu maior crescimento, mesmo na presença de Al. Na tentativa de verificar se tal hipótese era verdadeira, calculou-se, pelo menos em termos médios, a capacidade das plantas das duas cultivares de consumir ou liberar prótons, por unidade de massa de matéria seca radicular (Tabela 2).

Exceto nas soluções contendo o $\mathrm{N}$ exclusivamente na forma nítrica, observou-se liberação de prótons, que foi crescente com o aumento na concentração de $\mathrm{NH}_{4}{ }^{+}$ na solução nutritiva, nas duas cultivares (Tabela 2). Na presença de $\mathrm{N}$ exclusivamente na forma $\mathrm{NO}_{3}{ }^{-}$, a cultivar tolerante consumiu mais prótons do que a cultivar sensível, mas na presença de $\mathrm{NH}_{4}{ }^{+}$, qualquer que fosse a proporção $\mathrm{NO}_{3}{ }^{-} / \mathrm{NH}_{4}{ }^{+}$na solução nutritiva, a cultivar sensível liberou mais prótons para a solução nutritiva, por grama de matéria seca, por dia. O Al alterou o balanço de prótons e, provavelmente, a atividade

Tabela 2. Efeito do Al sobre a exsudação de prótons por duas cultivares de arroz, para soluções nutritivas com diferentes proporções de $\mathrm{NO}_{3}{ }^{-} / \mathrm{NH}_{4}{ }^{+(1)}$.

\begin{tabular}{cccccc}
\hline Proporção & \multicolumn{3}{c}{ Taxa diária de exsudação de prótons $\left(\mu \mathrm{mol} \mathrm{g}{ }^{-1} \text { dia }^{-1}\right)^{(2)}$} \\
\cline { 2 - 3 } $\mathrm{NO}_{3}{ }^{-} / \mathrm{NH}_{4}{ }^{+}$ & \multicolumn{2}{c}{ Fernandes (tolerante) } & & \multicolumn{2}{c}{ Maravilha $($ sensível) } \\
\cline { 2 - 3 } \cline { 5 - 6 } \cline { 5 - 6 } & $\mathrm{Al}=0 \mathrm{mM}$ & $\mathrm{Al}=1,5 \mathrm{mM}$ & & $\mathrm{Al}=0 \mathrm{mM}$ & $\mathrm{Al}=1,5 \mathrm{mM}$ \\
\hline $100 / 0$ & $-381,1 \mathrm{Db}$ & $-59,4 \mathrm{Da}$ & & $-294,8 \mathrm{Db}$ & $-35,3 \mathrm{Da}$ \\
$87,5 / 12,5$ & $271,3 \mathrm{Ca}$ & $42,2 \mathrm{Cb}$ & & $328,6 \mathrm{Ca}$ & $201,2 \mathrm{Cb}$ \\
$50 / 50$ & $326,1 \mathrm{Ba}$ & $68,9 \mathrm{Bb}$ & & $425,4 \mathrm{Ba}$ & $323,6 \mathrm{Bb}$ \\
$0 / 100$ & $726,0 \mathrm{Aa}$ & $192,1 \mathrm{Ab}$ & & $1.041,8 \mathrm{Aa}$ & $422,7 \mathrm{Ab}$ \\
\hline
\end{tabular}

(1)Médias, nas colunas, seguidas pela mesma letra maiúscula, para cada nível de $\mathrm{Al}$, e minúscula entre níveis de $\mathrm{Al}$, para cada proporção de $\mathrm{NO}_{3}{ }^{-} / \mathrm{NH}_{4}{ }^{+}$, para a mesma cultivar, não diferem entre si a $5 \%$ de probabilidade, pelo teste de Tukey. ${ }^{(2)}$ Prótons $\left(\mathrm{H}^{+}\right)$exsudados (valores positivos) ou absorvidos (valores negativos) da solução nutritiva. dos transportadores de $\mathrm{N}$ nas plantas alterando, conseqüentemente, a taxa de exsudação (ou consumo) de prótons nas soluções de cultivo, independentemente da proporção de $\mathrm{NO}_{3}{ }^{-} / \mathrm{NH}_{4}{ }^{+}$. Em soluções nutritivas contendo $\mathrm{N}$ exclusivamente na forma de $\mathrm{NO}_{3}{ }^{-}$, observouse redução de $84 \%$ e $88 \%$ na capacidade das plantas de alcalinizar o meio de cultivo, pelas cultivares tolerante e sensível, respectivamente. Em soluções nutritivas em que o $\mathrm{NH}_{4}{ }^{+}$estava presente, qualquer que fosse sua proporção, o Al reduziu, em média, em $79 \%$ e $41 \%$ a capacidade das plantas de acidificar o meio de cultivo, pelas cultivares tolerante e sensível, respectivamente. A análise do efeito do $\mathrm{Al}$ em soluções mistas com $\mathrm{NO}_{3}{ }^{-}$ e $\mathrm{NH}_{4}{ }^{+}$é mais complexa, uma vez que as plantas poderiam estar utilizando os dois sistemas de transporte ao mesmo tempo. Em solução nutritiva contendo o $\mathrm{N}$ exclusivamente na forma de $\mathrm{NO}_{3}{ }^{-}$, provavelmente, apenas o simporte $\mathrm{H}^{+} / \mathrm{NO}_{3}{ }^{-}$estava mediando a absorção desse ânion e, em tal condição, estima-se que a cultivar tolerante estava retornando ao simplasma (juntamente com o $\mathrm{N}$ absorvido) $29,3 \%$ e $68,3 \%$ mais prótons do que a cultivar sensível, na ausência e na presença de $\mathrm{Al}$, respectivamente. Isto indica que a cultivar tolerante foi capaz de manter, na solução nutritiva, menos prótons e, portanto, $\mathrm{pH}$ mais elevado, que pode reduzir a absorção do $\mathrm{Al}$ (Tabela 1). Em solução nutritiva contendo apenas $\mathrm{NH}_{4}{ }^{+}$como fonte de $\mathrm{N}$, a cultivar sensível deixou de retornar ao simplasma 43,5\% e 120\% menos prótons do que a tolerante, na ausência e na presença de Al, respectivamente. Isto, novamente, indica que a cultivar sensível deixou, na solução nutritiva, mais prótons, que facilita a absorção de Al (Tabela 1). As diferenças genotípicas das duas cultivares de arroz utilizadas no presente experimento, expressas em suas capacidades de modificar o $\mathrm{pH}$ do meio de cultivo, ao contrário do que sugerem Antunes \& Nunes (1997) para triticale, e Cambraia et al. (1987) para sorgo, parecem ser relevantes para a tolerância ao alumínio.

Tudo indica que o Al, realmente, afeta a aquisição de $\mathrm{N}$ pelas plantas e, conseqüentemente, modifica o balanço de prótons das soluções nutritivas. Com qualquer forma de fornecimento do N, a cultivar tolerante mostrou-se capaz de ajustar seu balanço de prótons de modo mais favorável, no que concerne à toxidez de $\mathrm{Al}$, do que a sensível.

\section{Conclusões}

1. As variáveis de crescimento estudadas sofrem redução na presença de $\mathrm{Al}$, redução essa que se acentua com o aumento na proporção relativa de $\mathrm{NH}_{4}{ }^{+}$na solução nutritiva. 
2. Os teores de $\mathrm{Al}$ são maiores nas plantas cultivadas em solução nutritiva com $\mathrm{NH}_{4}{ }^{+}$e aumentam com o acréscimo na concentração desse íon na solução nutritiva.

3. A cultivar Fernandes (tolerante) apresenta menor teor de $\mathrm{Al}$, independentemente da proporção $\mathrm{NO}_{3}{ }^{-} /$ $\mathrm{NH}_{4}{ }^{+}$, e sofre com menor intensidade os efeitos tóxicos desse cátion.

4. A cultivar Fernandes (tolerante) produz raízes mais compridas e numerosas do que a Maravilha (sensível), na presença ou na ausência de $\mathrm{Al}$ e, em conseqüência, consome sempre mais $\mathrm{H}^{+}$das soluções nutritivas, com $\mathrm{N}$ exclusivamente na forma de $\mathrm{NO}_{3}{ }^{-}$, e menos $\mathrm{H}^{+}$nas soluções com o $\mathrm{N}$ exclusivamente na forma de $\mathrm{NH}_{4}{ }^{+}$, ou em diferentes proporções de $\mathrm{NO}_{3}{ }^{-} / \mathrm{NH}_{4}{ }^{+}$.

5. A cultivar Fernandes (tolerante) é capaz de ajustar mais eficientemente seu balanço de prótons, de modo a absorver menos $\mathrm{Al}$, e a tolerar mais a presença desse cátion na solução nutritiva, do que a Maravilha (sensível).

\section{Agradecimentos}

Ao CNPq, pelas bolsas concedidas ao segundo e terceiro autores; ao Dr. Cléber Morais Guimarães, da Embrapa Arroz e Feijão, pelo fornecimento das sementes.

\section{Referências}

ANTUNES, A.M.G.; NUNES, M.A. Effects of aluminum on nutrients solution $\mathrm{pH}$ and nitrate/ammonium uptake by triticale. Journal of Plant Nutrition, v.20, p.1391-1401, 1997.

BERNARDO, L.M.; CLARK, R.B.; MARANVILLE, J.W. Nitrate/ ammonium ratio effects on nutrient solution $\mathrm{pH}$, dry matter yield, and nitrogen uptake of sorghum. Journal of Plant Nutrition, v.7, p.1389-1400, 1984.

CAMBRAIA, J.; CAMBRAIA, M.C. Avaliação de híbridos de milho quanto à tolerância ao alumínio, em solução nutritiva. Revista Ceres, v.42, p.297-307, 1995.

CAMBRAIA, J.; CHANDIAS, J.E.T.; ESTEVÃO, M.M.; SANT’ANNA, R. Efeito do alumínio sobre o balanço iônico e sobre a capacidade das plantas de sorgo para modificar o $\mathrm{pH}$ das soluções nutritivas. Revista Ceres, v.34, p.284-292, 1987.

CAMBRAIA, J.; SILVA, M.A.; CANO, M.A.O.; SANT’ANNA, R. Método simples para a avaliação de cultivares de sorgo quanto à tolerância ao alumínio. Revista Brasileira de Fisiologia Vegetal, v.3, p.87-95, 1991.

CLARK, J. Characterization of phosphatase of intact maize roots. Journal of Agricultural and Food Chemistry, v.23, p.458-460, 1975.
DEGENHARDT, J.; LARSEN, P.B.; HOWELL, S.H.; KOCHIAN, L.V. Aluminum resistance in the arabidopsis mutant alr-104 is caused by an aluminum-induced increase in rhizosphere $\mathrm{pH}$. Plant Physiology, v.117, p.19-27, 1998.

FAGERIA, N.K.; CARVALHO, J.R.P. Influence of aluminum in nutrient solutions on chemical composition in upland rice cultivars. Plant and Soil, v.69, p.31-44, 1982.

FAGERIA, N.K.; WRIGHT, R.J.; BALIGAR, V.C. Rice cultivar response to aluminum in nutrient solution. Communication in Soil Science and Plant Analysis, v.19, p.1133-1142, 1988.

FLEMING, A.L. Ammonium uptake by wheat varieties differing in Al tolerance. Agronomy Journal, v.75, p.726-730, 1983.

GANESAN, K.; SANKARANARAYANAN, C.; BALAKUMAR, T. Physiological basis of differential aluminum tolerance in rice genotypes. Communication in Soil Science and Plant Analysis, v.24, p.2179-2191, 1993.

GRAUER, U.E.; HORST, W.J. Effect of pH and nitrogen source on aluminium tolerance of rye (Secale cereale L.) and yellow lupin (Lupinus luteus L.). Plant and Soil, v.127. p.13-21, 1990.

MARSCHNER, H. Mineral nutrition of higher plants. $2^{\text {nd }}$ ed. London: Academic Press, 1995. 889p.

SANTAMARIA, P.; ELIA, A.; SERIO, F.; GONNELLA, M.; PARENTE, A. Comparison between nitrate and ammonium nutrition in fennel, celery, and swiss chard. Journal of Plant Nutrition, v.22, p.1091-1106, 1999.

SILVA, I.R.; SMYTH, T.J.; BARROS, N.F.; NOVAIS, R.F. Physiological aspects of aluminum toxicity and tolerance in plants. Tópicos em Ciência do Solo, v.2, p.277-335, 2002.

SIVAGURU, M.; PALIWAL, K. Differential aluminum tolerance in some tropical rice cultivars. II. Mechanism of aluminum tolerance. Journal of Plant Nutrition, v.16, p.1717-1732, 1993.

SMITH, R.L.; MILLS, H.A.; HOVELAND, C.S.; HANNA, W.W. Influence of ammonium/nitrate ratios on the growth and nitrogen uptake of pearl millet. Journal of Plant Nutrition, v.13, p.541553, 1990.

TAYLOR, G.J.; FOY, C.D. Mechanisms of aluminum tolerance in Triticum aestivum (wheat): IV. The role of ammonium and nitrate nutrition. Canadian Journal of Botany, v.63, p.2181-2186, 1985.

TISCHNER, R. Nitrate uptake and reduction in higher and lower plants. Plant, Cell and Environment, v.23, p.1005-1024, 2000.

TRAORE, A.; MARANVILLE, W. Effect of nitrate/ammonium ratio on biomass production, nitrogen accumulation, and use efficiency in sorghums of different origin. Journal of Plant Nutrition, v.22, p.813-825, 1999.

WANG, C.; WOOD, F.A. A modified aluminon reagent for the determination of aluminum after $\mathrm{HNO}_{3}-\mathrm{H}_{2} \mathrm{SO}_{4}$ digestion. Canadian Journal of Soil Science, v.53, p.237-239, 1973.

Recebido em 13 de agosto de 2004 e aprovado em 24 de dezembro de 2004 